\title{
Coexistence of early microinvasive endometrioid adenocarcinoma and CIN3 in the uterine cervix in a 32-year-old Japanese woman
}

Tadashi Terada ${ }^{1,2}$

\begin{abstract}
Simultaneous occurrence of early microinvasive endometrioid adenocarcinoma (EMEA) and CIN 3 in the uterine cervix is very rare in Japan. A 32-year-old Japanese woman was pointed out to have atypical cells in the cervical cytology. Colposcopic examination revealed irregular lesions in the cervix, and a biopsy showed simultaneous EMEA and CIN3. The EMEA was grade I and CIN3 corresponded to severe dysplasia/carcinoma in situ. Hysterectomy and lymph nodes dissection were performed. Grossly, mucosal irregularity and erosion were seen in the cervix. No tumor formation was recognized. The cervix was examined by serial sections. Microscopically, there were a tiny adenocarcinoma $(0.5 \mathrm{~cm}$ in diameter and $0.3 \mathrm{~cm}$ in depth) and broad areas of CIN3. The adenocarcinoma was EMEA without mucins. The EMEA was FIGO stage 1A1. Immunohistochemically, the EMEA was positive for pancytokeratins (AE1/2 +++, CAM5.2 ++), cytokeratin (CK) 34ßE12 +, CK5/6 +, CK7 +, CK18 +++, CK19 ++, CA19-9 +, CA125 +++, p53 +, ER +++, PgR +++, while it was negative for CK8, CK14, CK20, EMA, vimentin, CEA, desmin, smooth muscle actin, p63, chromogranin, synaptophysin, CD56, CD68, HER2/neu, MUC1, MUC2, MUC5AC, and MUC6. The CIN 3 was positive for pancytokeratins (AE1/2 +++, CAM5.2 +), cytokeratin (CK) 34ßE12 +++, CK5/6 +++, CK7 +, EMA, CA19-9 +, CA125 +, p53 +, p63 +++, ER +++, and MUC1 +, while it was negative for CK8, CK14, CK18, CK19, CK20, vimentin, CEA, desmin, smooth muscle actin, chromogranin, synaptophysin, CD56, CD68, PgR, HER2/neu, MUC2, MUC5AC and MUC6. The lymph nodes showed no metastatic lesions (0/34). In conclusion, the author reported a rare case of simultaneous EMEA and CIN 3 with extensive immunohistochemical findings.
\end{abstract}

Virtual Slides: The virtual slide(s) for this article can be found here: http://www.diagnosticpathology.diagnomx.eu/ vs/2229343835388013.

Keywords: early microinvasive adenocarcinoma, CIN3, uterine cervix, histopathology, immunohistochemistry

\section{Introduction}

Malignant neoplasms of the uterine cervix are frequent in Japan. Most of them are squamous cell carcinoma and cervical intraepithelial neoplasm (CIN), and adenocarcinoma and its precursor lesions are rare in Japan.

Adenocarcinoma of the uterine cervix was classified into adenocarcinoma NOS, mucinous adenocarcinoma, endometrioid adenocarcinoma, clear cell adenocarcinoma, serous adenocarcinoma mesonephric adenocarcinoma, early microinvasive adenocarcinoma, and adenocarcinoma in situ [1]. The mucinous adenocarcinoma was subclassified

Correspondence: piyo0111jp@yahoo.co.jp

${ }^{1}$ Department of Pathology, Shizuoka City Shimizu Hospital, Shizuoka, Japan Full list of author information is available at the end of the article into endocervical, intestinal, signet-ring cell, minimal deviation, and villoglandular subtypes [1]. Glandular dysplasia equivalent to squamous dysplasia is also present.

More than $90 \%$ of uterine tumorous lesions are squamous cell carcinoma and its precursor lesions. The precursor lesions of the squamous cell carcinoma had traditionally been called dysplasia (mild, moderate, and severe) and carcinoma in situ (CIS). Recently, they have been termed as cervical intraepithelial neoplasm (CIN) or squamous intraepithelial lesions (SIL) [1]. CIN is categorized as CIN 1-3, and SIL as low grade SIL (CIN 1) and high grade SIL(CIN2-3/CIS) (HGSIL) [1]. These lesions are known to be frequently associated with sexual intercourses and human papilloma virus (HPV) infection [1]. 
According to WHO [1], adenocarcinoma is associated with CIN in about $40 \%$ of cases. However, Brown et al [2] reported that only 1 case of adenocarcinoma was found in 105 cases of CIN 3. Therefore, the coexistence of adenocarcinoma and CIN 3 is very rare. The author herein reports a case of simultaneous early microinvasive endometrioid adenocarcinomna (EMEC) and CIN 3 in a young Japanese woman.

\section{Case report}

A 32-year-old Japanese woman was found to have atypical cells in the cervical cytology at routine check. Colposcopic examination revealed irregular lesions in the cervix. A biopsy revealed simultaneous adenocarcinoma (Figure 1) and CIN3 (Figure 2). The adenocarcinoma have grade I atypia and CIN3 corresponded to severe dysplasia/carcinoma in situ.

Hysterectomy and lymph nodes dissection were performed. Grossly, mucosal irregularity and erosion were seen in the cervix (Figure 3). No tumor formation was recognized. The cervix was examined by serial sections. There were a tiny adenocarcinoma $(0.5 \mathrm{~cm}$ in diameter and $0.3 \mathrm{~cm}$ in depth) (Figure 4 ) and broad areas of CIN3 (Figure 5). Both lesions are separate, and no merges between both lesions were seen. The adenocarcinoma was EMEA without mucins, as revealed by negative alcian blue/PAS staining. The lymph nodes were negative for metastasis $(0 / 34)$. No metastatic foci were found in the body. The EMEA was FIGO stage $1 \mathrm{~A} 1$.

An immunohistochemical study was performed with the use of DAKO Envision method (Dako Corp., Glostrup, Denmark), as described previously [3-7]. The immunohistochemical results are shown in Table 1. Immunohistochemically, the EMEA was positive for pancytokeratins (AE1/2+++, CAM5.2 ++), cytokeratin (CK) $34 \beta \mathrm{BE} 12+, \mathrm{CK} 5 / 6+$, CK7 +, CK18 +++(Figure 6), CK19 ++, CA19-9 +, CA125 +++ (Figure 7), p53 +, ER +++ , PgR +++ , while it was negative for CK8, CK14, CK20, EMA, vimentin, CEA, desmin, smooth muscle actin, p63, chromogranin, synaptophysin, CD56, CD68, HER2/neu, MUC1, MUC2, MUC5AC, and MUC6. The CIN 3 was positive for pancytokeratins (AE1/2 +++ , CAM5.2 +), cytokeratin (CK) 34ßE12 +++ (Figure 8), CK5/6 +++, CK7 +, EMA, CA19-9 +, CA125 +, p53 +, p63 +++ (Figure 9), ER +++, and MUC1 + (Figure 10), while it was negative for CK8, CK14, CK18, CK19, CK20, vimentin, CEA, desmin, smooth muscle actin, chromogranin, synaptophysin, CD56, CD68, PgR, HER2/ neu, MUC2, MUC5AC, and MUC6. The patient is now free from tumor 4 months after the operation.

\section{Discussion}

The adenocarcinoma in the present case was early microinvasive with width of $0.5 \mathrm{~cm}$ and depth of 0.3 $\mathrm{cm}$. Histologically, the adenocarcinoma resembled

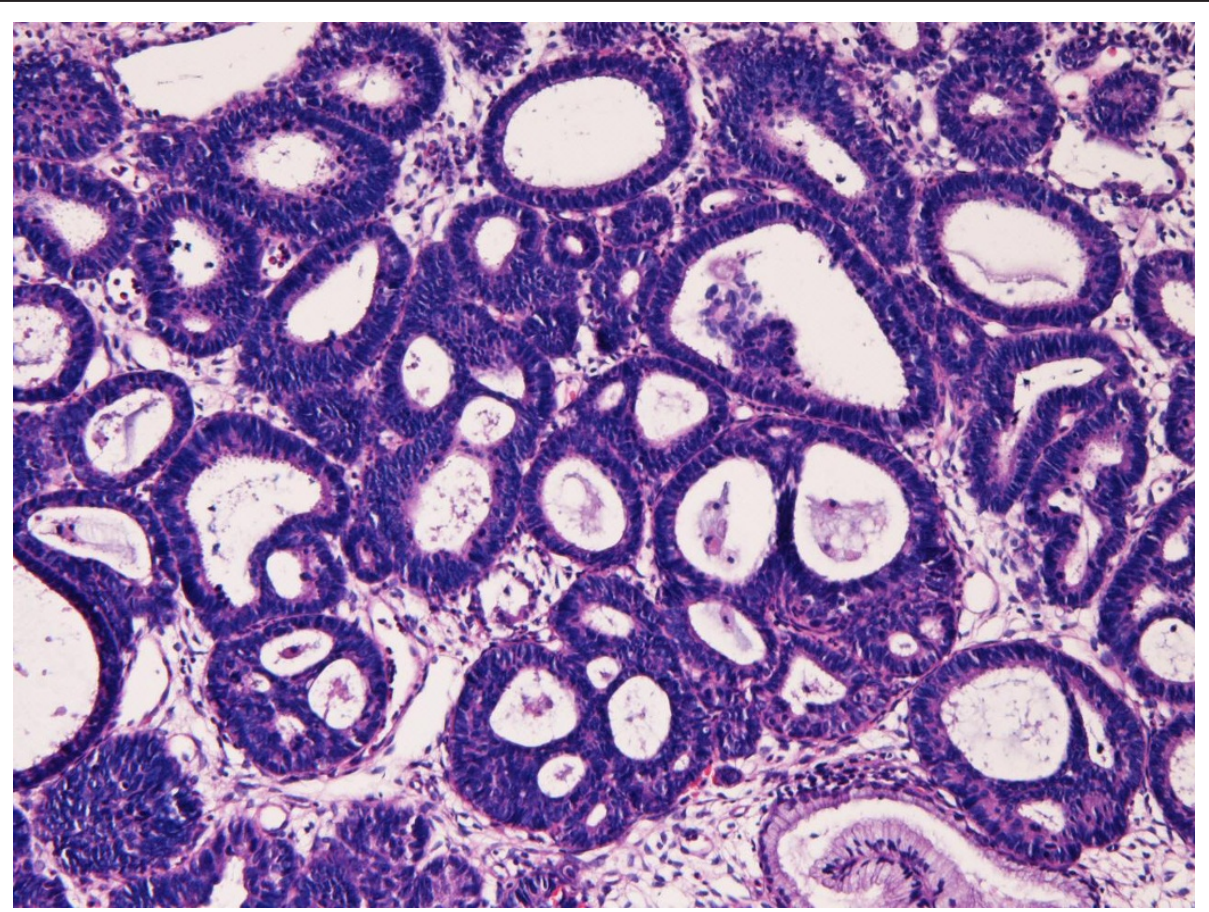

Figure 1 Biopsy of uterine cervix. The biopsy shows atypical glands with hyperchromatic nuclei and with structural atypia. This lesion is regarded as adenocarcinoma. $\mathrm{HE}, \times 100$. 


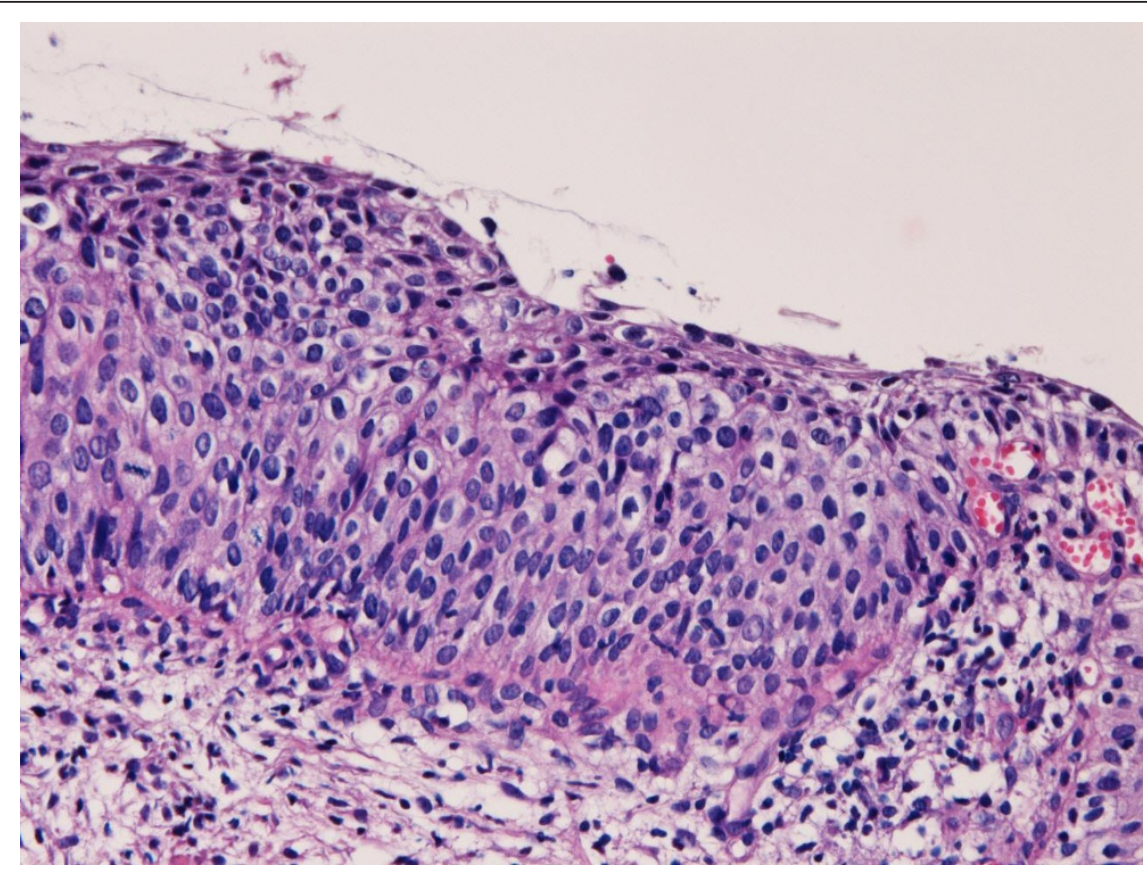

Figure 2 Biopsy of uterine cervix. The biopsy shows atypical squamous lesion. The atypical cells showed hyperchromatic nuclei and occupy all layers. The polarity and stratification are lost. The lesion was regarded as CIN3. HE, $\times 100$.

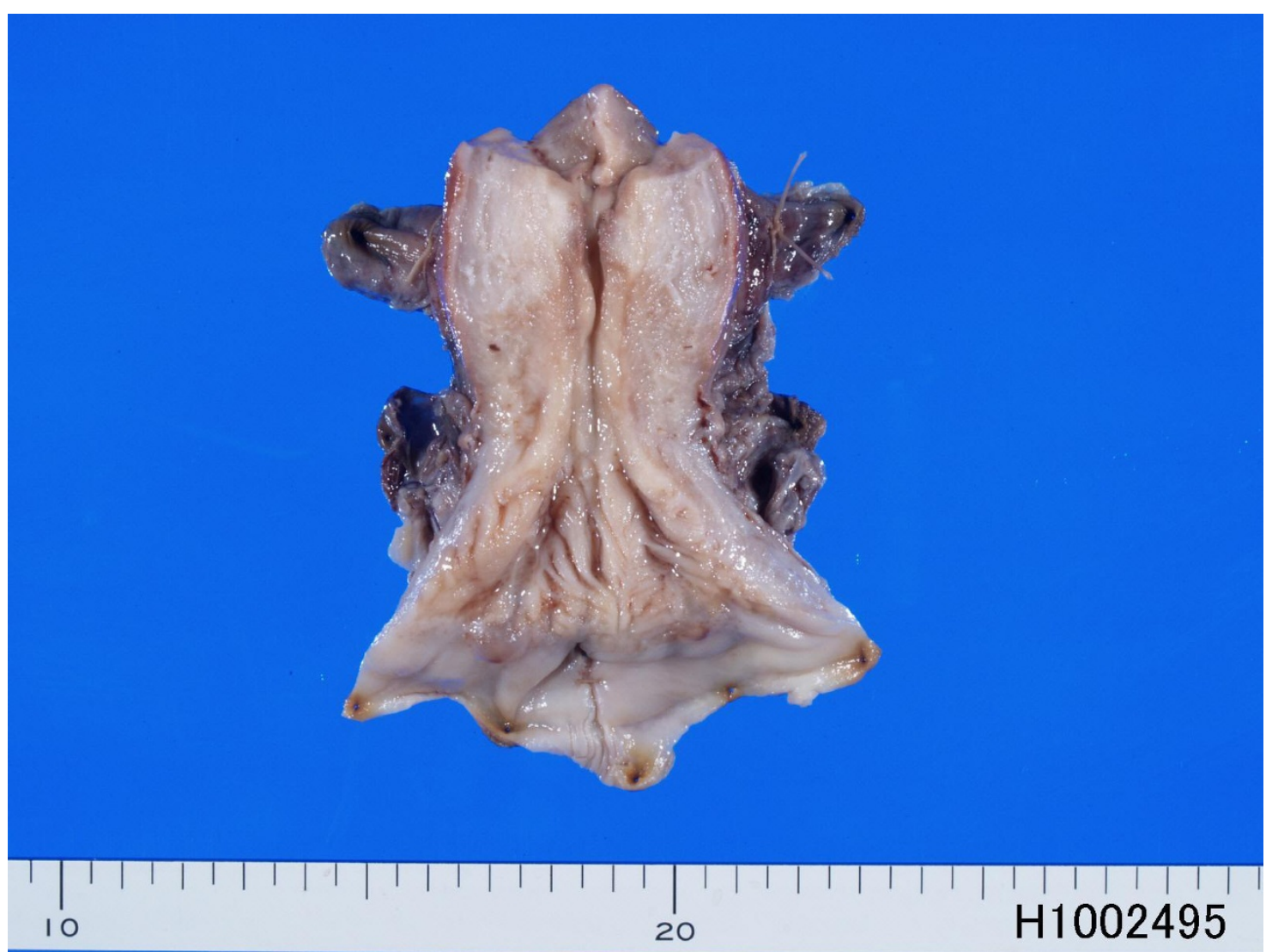

Figure 3 Gross features of resected uterus. The cervix shows irregularity and erosions. No apparent tumor is seen. 


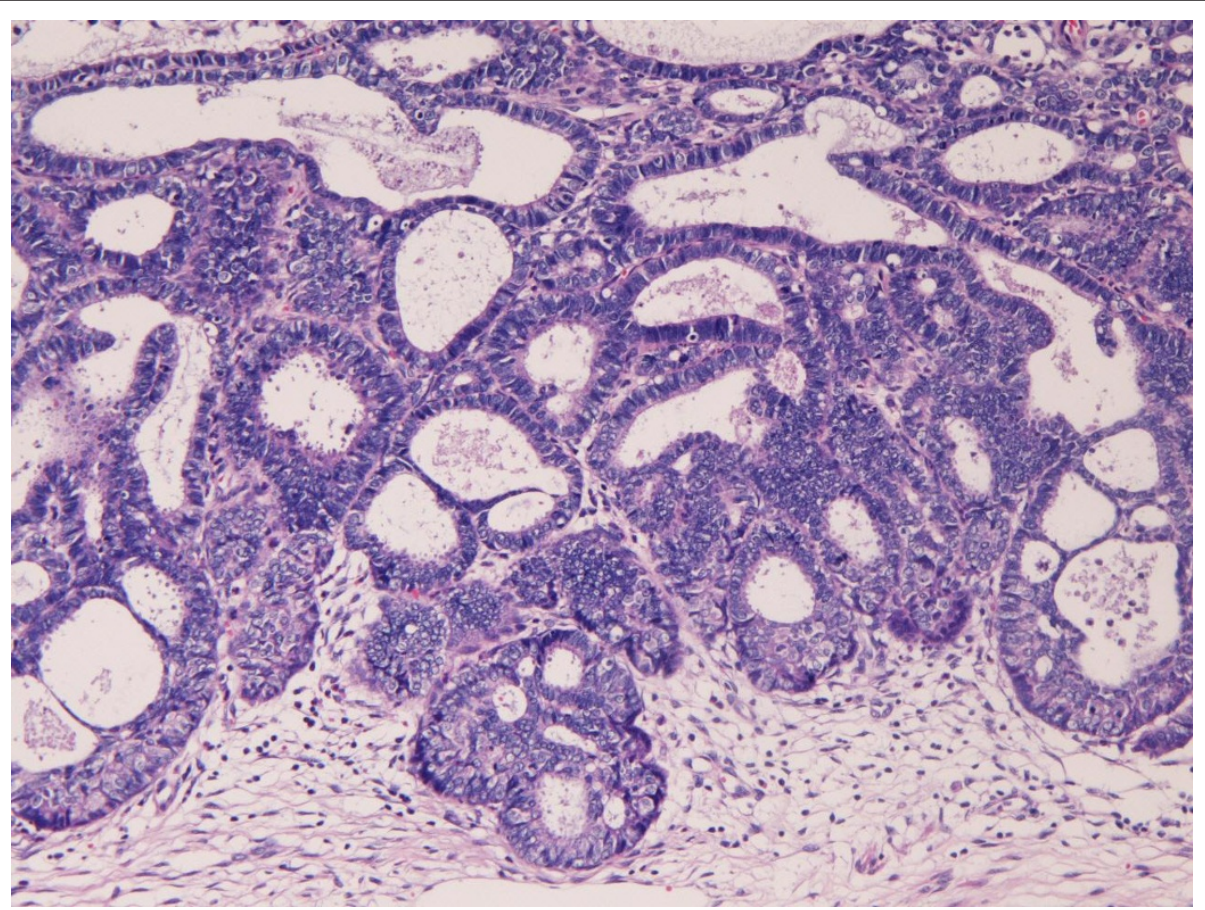

Figure 4 Microscopic features of the resected uterus. The adenocarcinoma component The adenocarcinoma is endometrioid adenocarcinoma. The carcinoma is small with mild invasion. HE $\times 50$.

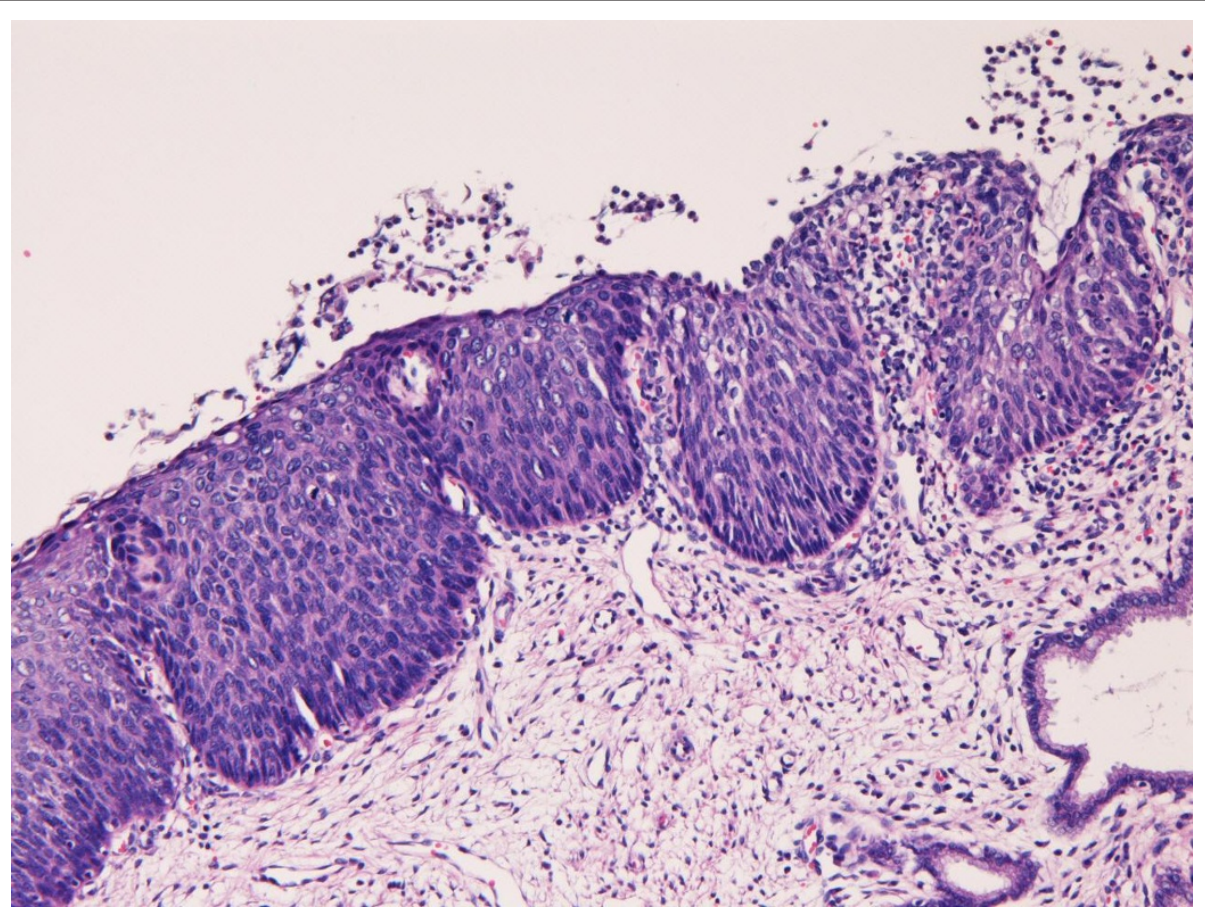

Figure 5 Microscopic features of the resected uterus. The CIN3 component. CIN3 without invasion is seen. HE $\times 50$. 
Table 1 Immunohistochemical reagents and results

\begin{tabular}{|c|c|c|c|c|}
\hline \multirow[b]{2}{*}{ Antigens } & \multirow[b]{2}{*}{ Antibodies (clone) } & \multirow[b]{2}{*}{ Sources } & \multicolumn{2}{|l|}{ Results } \\
\hline & & & EMEA. & CIN3 \\
\hline Pancytokeratin & $\mathrm{AE} 1 / 3$ & Dako Corp. Glostrup, Denmark & +++ & +++ \\
\hline Pancytokeratin & CAM5.2 & Beckton-Dickinson, CA, USA & ++ & + \\
\hline HMWCK & $34 \beta \mathrm{E} 12$ & Dako & + & +++ \\
\hline CK5/6 & D5/16 & Dako & + & +++ \\
\hline CK7 & N1626 & Dako & + & + \\
\hline CK8 & DC10 & Dako & - & - \\
\hline CK14 & LL002 & Novocastra, Newcastle upon type, UK & - & - \\
\hline CK 18 & DC10 & Dako & +++ & - \\
\hline CK 19 & RCK 108 & Progen, Heidelberg, Germany & ++ & - \\
\hline CK 20 & K20.8 & Dako & - & - \\
\hline EMA & E29 & Dako & - & + \\
\hline Vimentin & Vim 3B4 & Dako & - & - \\
\hline CEA & polyclonal & Dako & - & - \\
\hline CA19-9 & NS19-9 & TBF Lab, Tokyo Japan & + & + \\
\hline CA125 & NS125 & TFB Lab & +++ & + \\
\hline Desmin & D33 & Dako & - & - \\
\hline ASMA & $1 \mathrm{~A} 4$ & Dako & - & - \\
\hline p53 protein & DO-7 & Dako & + & + \\
\hline p63 & $4 \mathrm{~A} 4$ & Dako & - & +++ \\
\hline Chromogranin & DAK-A3 & Dako & - & - \\
\hline Synaptophysin & Polyconal & Dako & - & - \\
\hline CD56 & UJ13A & Dako & - & - \\
\hline CD68 & $\mathrm{KP}-1$ & Dako & - & - \\
\hline ER & M7047 & Dako & +++ & +++ \\
\hline $\mathrm{PgR}$ & $1 \mathrm{~A} 6$ & Novocastra & +++ & - \\
\hline HER2/neu & CB11 & Ventana Japan, Tokyo & - & - \\
\hline MUC1 & Ma695 & Novocastra & - & + \\
\hline MUC2 & CCp58 & Novocastra & - & - \\
\hline MUC5AC & $\mathrm{CLH} 2$ & Novocastra & - & - \\
\hline MUC6 & CLH5 & Novocastra & - & - \\
\hline
\end{tabular}

+++ , strongly positive. ++ , moderately positive. + , mildly positive. -, negative. EMEA, early microinvasive endometrioid adenocarcinoma. CIN3, cervical intraepithelial neoplasm grade 3. HMWCK, high molecular weight cytokeratin. CK, cytokeratin. EMA, epithelial membrane antigen. CEA, carcinoembryonic antigen. CA19-9, carbohydrate antigen 19-9. CA125, carbohydrate antigen 125. ASMA, $\alpha$-smooth muscle antigen. ER, estrogen receptor. PgR, progesterone receptor.

endometrial endometrioid adenocarcinoma (grade 1), and negative for mucins. Therefore, the adenocarcinoma was EMEA [1]. The diagnosis of CIN 3 is apparent in the present case [1].

Squamous cell carcinoma and adenocarcinoma can coexist in the uterine cervix [8-13]; there are several case reports of this in the English literature [8-13]. From the standpoint of CIN, Brown et al [2] reported that 16 cases of cervical glandular atypia and only 1 case adenocarcinoma in situ were found in 105 cases of CIN 3. From the standpoint of cervical adenocarcinoma, Maier and Norris [9] reported that 99 cases of CIN were detected in 230 cases of cervical adenocarcinoma. About half of such cases were CIN 2 or CIN 3 [9]. There is only one report that there is an association of adenocarcinoma in situ and cervical squamous cell carcinoma [10]. However, cases of simultaneous adenocarcinoma and CIN3 are very rare in the English literature [10,14-19]. In addition, cases of simultaneous early microinvasive adenocarcinoma and CIN3 are very rare $[17,18]$. Furthermore, cases of simultaneous endometrioid adenocarcinoima and CIN3 are extremely rare. Therefore, the present case of coexistence of EMEA and CIN3 appears extremely rare.

In the present study, the cervical lesion is typical CIN3, HGSIL, or CIS. No invasion was recognized. The histological features fulfill the diagnosis of CIN 3 [1]. The adenocarcinoma element in the present study was of EMEA negative for mucins [1]. In both elements, adequate structural and cytological atypia regarded as malignant was recognized. The positive immunoreaction of p53 protein in the present EMEA and CIN3 strongly suggests that both lesions in the present study are malignant. 


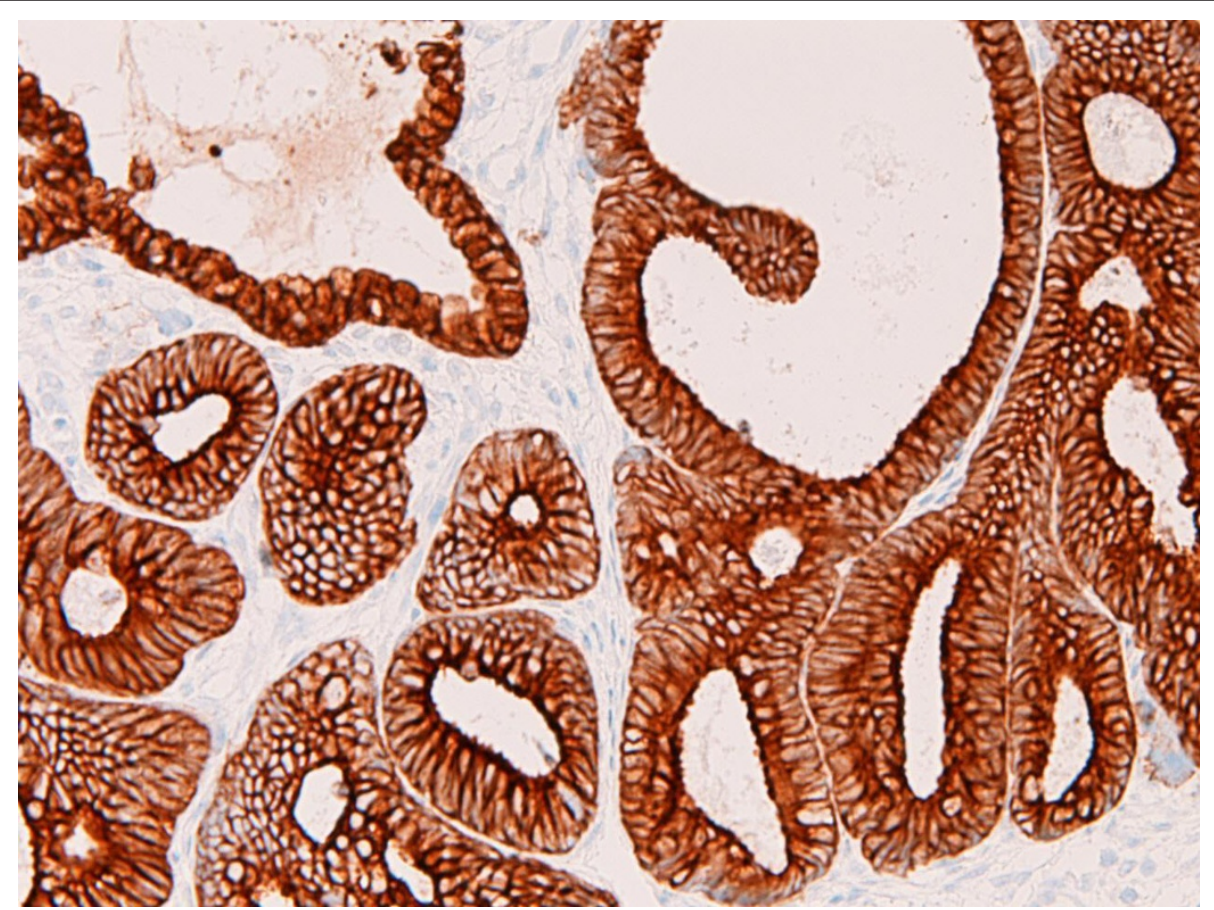

Figure 6 Immunohistochemical features. The adenocarcinoma component is strongly positive for cytokeratin 18. $\times 100$.

There have been few immunohistochemical studies in simultaneous squamous neoplasm and adenocarcinoma in the uterine cervix [10]. The present study extensively examined the immunoprofile of EMEA and CIN3. The EMEA and CIN3 showed different immunoprofile, suggesting that they are different neoplasms. The CK profiling of EMEA showed predominant presence of low molecular weight cytokeratin, while CIN3 high-molecular weight cytokeratin. Since squamous lesions express high-molecular weight CK while adenocarcinoma low-

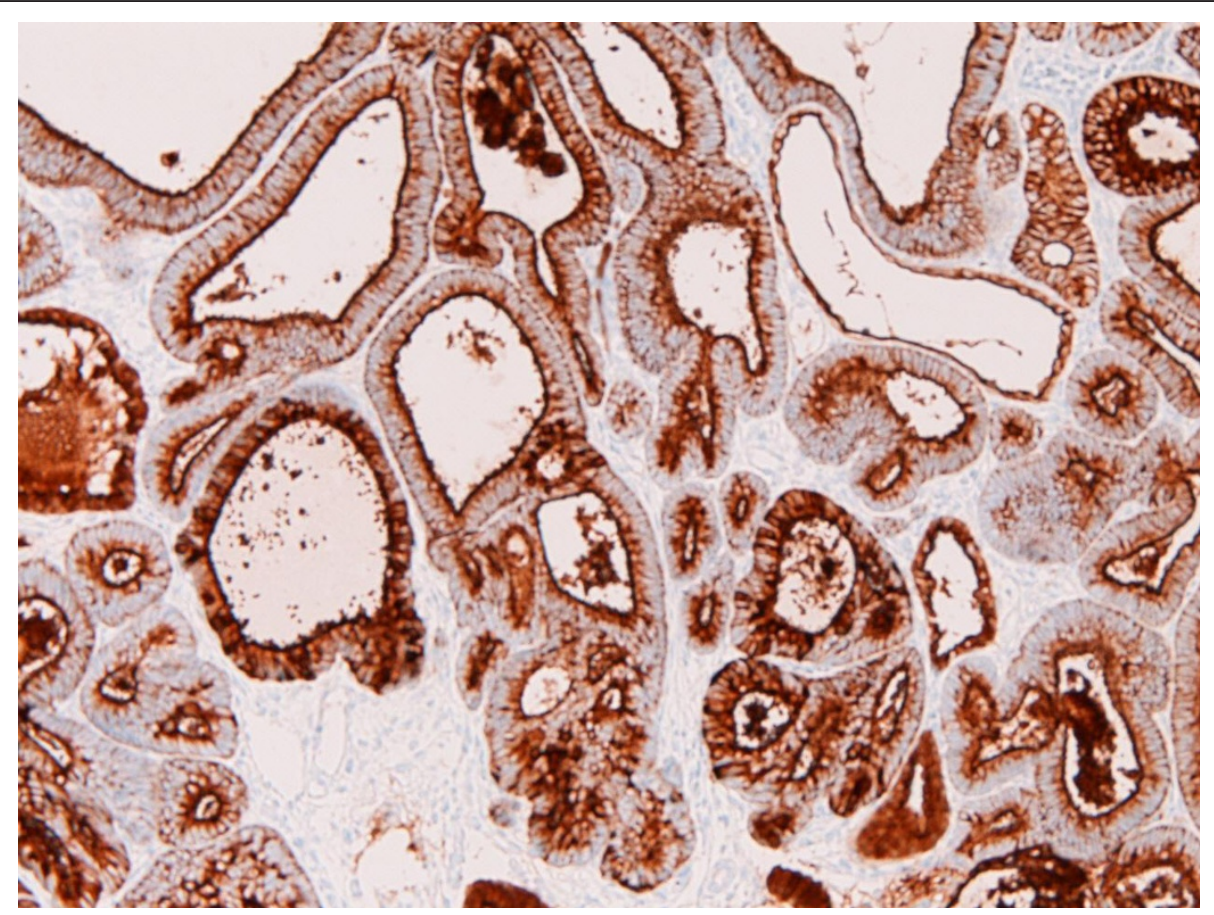

Figure $\mathbf{7}$ Immunohistochemical features. The adenocarcinoma component is strongly positive for CA125. ×100. 


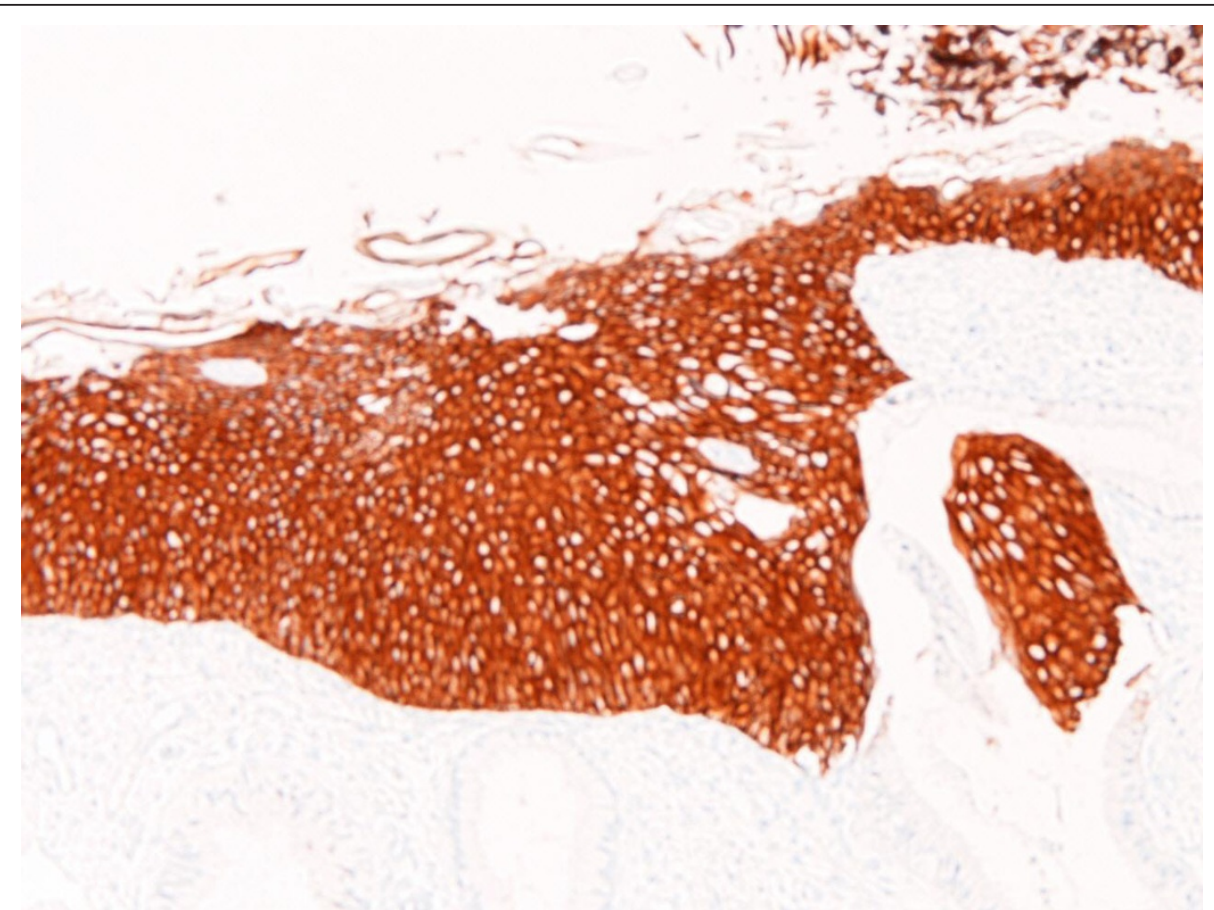

Figure 8 Immunohistochemical features. The CIN3 component was strongly positive for cytokeratin $34 \beta E 12 . \times 100$.

molecular weight cytokeratin [20], the present data indicate the CIN3 is squamous lesions and EMEA is adenocarcinoma. The presence of p63 in CIN3 but not in EMEA indicates that CIN3 was squamous lesion and EMEA is not, since p63 is known to be expressed in squamous lesions but not in adenocarcinoma lesions [21]. Both components were negative for CK14, CK17, and CK20, suggesting that these three CKs are not expressed in either lesion. EMA was positive in CIN3, but negative in EMEA, suggesting that EMA is

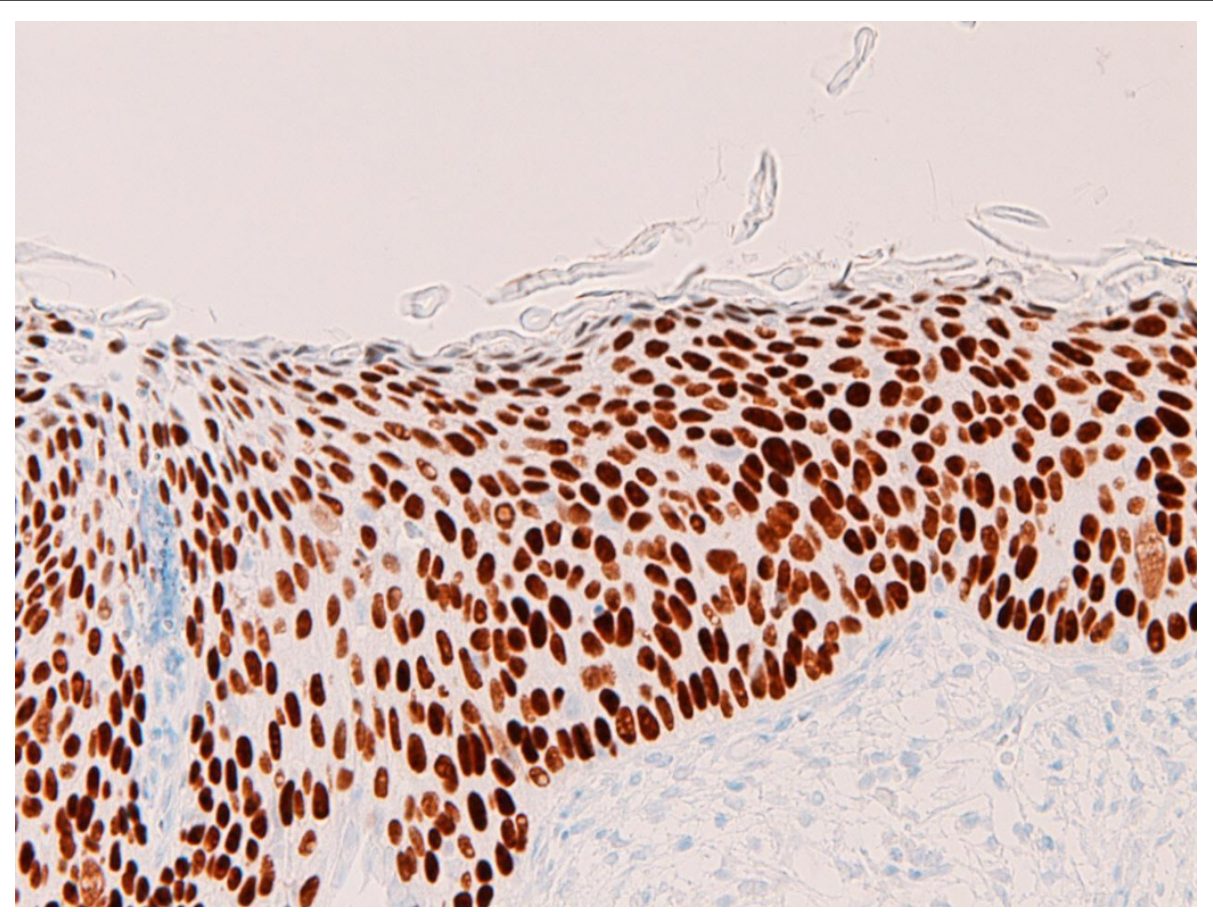

Figure 9 Immunohistochemical features. The CIN3 component was strongly positive for p63. $\times 100$ 


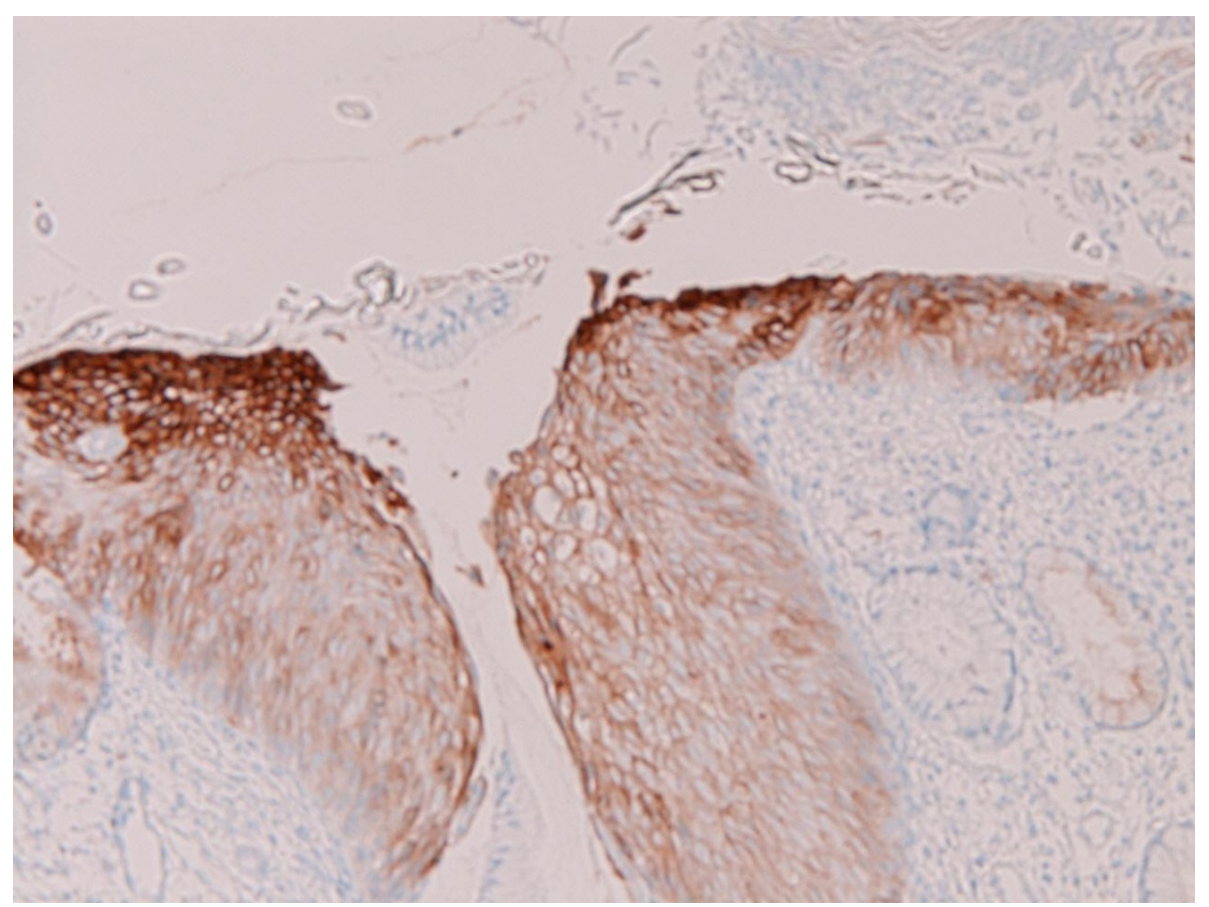

Figure 10 Immunohistochemical features. The CIN3 component was positive for MUC1. $\times 100$.

expressed only in CIN lesions. CA125 was strongly positive in EMA and mildly positive in CIN, suggesting that CA125 is mainly expressed in adenocarcinoma element rather than CIN. CA19-9 was mildly expressed in both EMEA and CIN3, suggesting that both lesions have a small amount of this carbohydrate antigen. p53 was positive in both components, suggesting that p53 gene is mutated in both components and that both components are malignant. Both components were strongly positive for ER, being compatible with the presence of ER of female genital organs. However, PgR was strongly positive for EMEA but negative in CIN3, suggesting the presence of PgR in EMEA, but not in CIN. Interestingly, MUC 1 was present in CIN3 but was absent in EMEA. MUC2, MUC5AC and MUC6 were negative in both components. MUC is mucin core protein gene product so that it was previously anticipated that MUCs are present in EMEA. These results indicate CIN3 may be positive for MUC1 and EMEA was negative for MUCs. Both elements were negative for chromogranin, synaptophysin, and CD56, indicating that both components do not show neuroendocrine features. Both components were negative for vimentin, desmin, and $\alpha$-smooth mucle actin, indicating that these three mesenchymal antigens were absent in EMEA and CIN3. CEA was negative for both components, suggesting that EMEA may be negative for CEA. CD68 was negative in both components, indicating no histiocytic differentiation. Both components were negative for HER2/neu, suggesting that both components were negative for this receptor.

In summary, the author presented a case of simultaneous EMEA and CIN3 of the uterine cervix in a 32year-old Japanese woman with an emphasis on immunohistochemical findings.

\section{Consent}

Written informed consent was obtained from the patient for publication of this case report and accompanying images. A copy of the written consent is available for review by the Editor-in-Chief of this journal.

\section{Author details}

'Department of Pathology, Shizuoka City Shimizu Hospital, Shizuoka, Japan. ²Department of Pathology, Shizuoka City Shimizu Hospital, Miyakami 1231 Shimizu-Ku, Shizuoka 424-8636, Japan.

\section{Competing interests}

The author declares that they have no competing interests.

Received: 9 April 2011 Accepted: 10 June 2011 Published: 10 June 2011

\section{References}

1. World Health Organization Classification of Tumours. Pathology and genetics of tumours of the breast and female genital organs. In Tumours of uterine cervix. Edited by: Tavassoli FA and Devilee. IARC press, Lyon; 2003:259-311.

2. Brown JL, Wells M: Cervical glandular atypia associated with squamous intraepithelial neoplasm: a premalignant lesion? I Clin Pathol 1986, 39:22-28. 
3. Terada T, Kawaguchi M, Furukawa K, Sekido Y, Osamura RY: Minute mixed ductal-endocrine carcinoma of the pancreas with predominant intraductal growth. Pathol Int 2002, 52:740-746.

4. Terada T, Kawaguchi M: Primary clear cell adenocarcinoma of the peritoneum. Tohoku J Exp Med 2005, 206:271-275.

5. Terada T, Taniguchi M: Intraductal oncocytic papillary neoplasm of the liver. Pathol Int 2004, 54:116-123.

6. Terada T, Moriki T: Monolobar ductal plate malformation disease of the liver. Pathol Int 2010, 60:407-412.

7. Terada TLarge cell neuroendocrine carcinoma with sarcomatous changes of the endometrium: a case report with immunohistochemical studies and molecular genetic study of KIT and PDGFRA. Pathol Res Pract 2010, 206:420-425.

8. Choo YC, Naylor B: Coexistance of squamous cell carcinoma and adenocarcinoma of the uterine cervix. Gynecol Oncol 1984, 17:168-174.

9. Maier RC, Norris HJ: Coexistance of cervical intraepithelial neoplasm with primary adenocarcinoma of the endocervix. Obstet Gynecol 1980, 56:361-364.

10. Terada T: Simultaneous squamous cell carcinoma in situ and adenocarcinoma in situ of the uterine cervix in a 36-year-old Japanese woman. Arch Gynecol Obstet 2010, 281:527-530.

11. Vasilijevic M, Stanimirovic B, Stanojevic D, Djukic M, Vasilijevic N: Primary double invasive cervical carcinoma, squamous cell carcinoma and adenocarcinoma: case report. Eur J Gynecol Oncol 2008, 29:411-413.

12. Goto K, Takeuchi Y, Yakihara A, Kosigi F: Synchronous invasive squamous cell carcinoma and clear cell adenocarcinoma of the uterine cervix: a different human papillomavirus status. Gynecol Oncol 2005, 97:976-979.

13. van Aspert-van Erp AJ, Smedts FM, Vooijs GP: Severe cervical glandular cell lesions with coexisting squamous cell lesions. Cancer 2004, 25:218-227.

14. Anastasiadis $P$, Koutlaki N, Tamiolakis D, Liberis $V$, Galazios $G$, Sivridis E: Invasive adenocarcinoma of mixed endocervical and clear cell types, associated with invasive squamous cell carcinoma of the cervix uteri: a case report. Eur J Gynecol Obstet 2000, 21:173-176.

15. Skopelitou A, Hadjiyannakis M: Enteric type villoglandular papillary adenocarcinoma of the uterine cervix associated with in situ squamous cell carcinoma: case report and review of the literature. Eur J Gynecol Obstet 1996, 17:309-314.

16. Lauchian SC, Penner DW: Simultaneous adenocarcinoma in situ and epidermoid carcinoma in situ: report of two cases. Cancer 1967 20:2250-2254.

17. Wolk BM, Kime W, Albites V: Simultaneous in situ squamous cell carcinoma and microinvasive adenocarcinoma of the cervix. Int J Gynecol Obstet 1981, 19:69-72.

18. Yamasaki M, Ueda G, Sato Y, Kobayashi Y, Kurachi K: Simultaneous squamous cell carcinoma in situ and early invasive adenocarcinoma of the uterine cervix. Acta Obstet Gynecol Jpn 1975, 22:6-9.

19. Toki T, Katayama Y, Motoyama T: Small cell neuroendocrine carcinoma of the uterine cervix associated with microinvasive squamous cell carcinoma and adenocarcinoma in situ. Pathol Int 1996, 46:520-525.

20. Smedts F, Ramaekers F, Troyanovsky S, Pruszczynski M, Link M, Lane B, Leich I, Schijf C: Keratin expression in cervical cancer. Am J Pathol 1992, 141:497-511.

21. Owens SR, Greenson JK: Immunohistochemical staining for p63 is usefu in the diagnosis of anal squamous cell carcinomas. Am J Surg Pathol 2007, 31:285-290.

\section{Submit your next manuscript to BioMed Central and take full advantage of:}

- Convenient online submission

- Thorough peer review

- No space constraints or color figure charges

- Immediate publication on acceptance

- Inclusion in PubMed, CAS, Scopus and Google Scholar

- Research which is freely available for redistribution

Submit your manuscript at www.biomedcentral.com/submit
Ciomed Central 